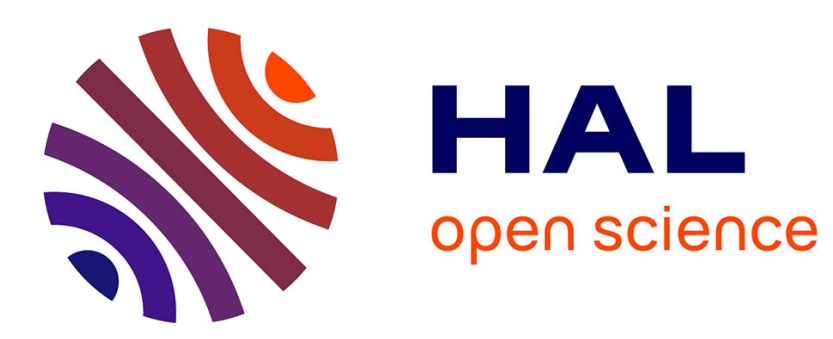

\title{
Les formes contemporaines de la biopolitique
}

Thibault Bossy, François Briatte

\section{To cite this version:}

Thibault Bossy, François Briatte. Les formes contemporaines de la biopolitique. Revue internationale de politique comparée, 2011, 18 (4), pp.7-12. 10.3917/ripc.184.0007 . hal-00675780

\section{HAL Id: hal-00675780 \\ https://hal.science/hal-00675780}

Submitted on 2 Mar 2013

HAL is a multi-disciplinary open access archive for the deposit and dissemination of scientific research documents, whether they are published or not. The documents may come from teaching and research institutions in France or abroad, or from public or private research centers.
L'archive ouverte pluridisciplinaire HAL, est destinée au dépôt et à la diffusion de documents scientifiques de niveau recherche, publiés ou non, émanant des établissements d'enseignement et de recherche français ou étrangers, des laboratoires publics ou privés.

\section{다(1) (2)}

Distributed under a Creative Commons Attribution - ShareAlike| 4.0 International 


\title{
Les formes contemporaines de la biopolitique*
}

\author{
Thibault Bossy et François Briatte
}

4 novembre 2011

Empruntée à Michel Foucault, la notion de «biopolitique » est couramment employée dans différentes disciplines des sciences sociales pour désigner l'évolution historique des politiques de santé publique sur la longue durée, et en particulier pour évoquer l'individualisation du risque dans les dispositifs de santé publique les plus récents. ${ }^{1}$ Elle peut s'employer similairement à d'autres, comme celle de processus de civilisation, empruntée à Norbert Elias, pour désigner les technologies employées par l'État à des fins de contrôle social, au niveau des individus comme à celui des populations. ${ }^{2}$ Un très grand nombre de travaux sociologiques consacrés à l'étude des phénomènes contemporains de « biomédicalisation $»^{3}$ en font aujourd'hui cet usage, non restrictif par ailleurs, comme le montrent les travaux usant des mêmes fondations intellectuelles pour développer une approche critique de la sécurité internationale. ${ }^{4} \mathrm{La}$ littérature scientifique montre ainsi qu'au cours des vingt dernières années, la notion de « biopolitique » chez Michel Foucault a dépassé le cercle des commentateurs de Foucault luimême : elle innerve désormais une pluralité de courants de recherche en sciences sociales, de la même manière que la notion de "discipline ». Ces deux pans de l'armature intellectuelle foucaldienne recouvrent un espace de réflexion plus vaste encore, constitué autour de la « gouvernementalité »" ${ }^{5}$ c'est-à-dire des technologies de subjectivation déployées par les autorités politiques dans leur quête de domination et de contrôle sur les autres agents sociaux.

\footnotetext{
${ }^{*}$ Revue internationale de politique comparée, à paraître. Les auteurs remercient Luc Berlivet et Pierre Lascoumes pour leur discussion des textes au $10^{\mathrm{e}}$ Congrès de 1'Association Française de Science Politique (Grenoble, septembre 2009), ainsi que l'ensemble des participants à la section thématique $\mathrm{n}^{\circ} 22$, qui donne son nom à ce dossier thématique. Merci également à Alexandre Hobeika pour sa relecture attentive d'une première version du texte.
}

${ }^{1}$ BUNTON R. et PETERSEN A. (dir.), Foucault, Health and Medicine, Londres : Routledge, 1997 ; JONES C. (dir.), Reassessing Foucault: Power, Medicine and the Body, Londres : Routledge, 1994 ; LEMKE T., Biopolitics: An Advanced Introduction, New York/Londres : New York University Press, 2011 ; LUPTON D., The Imperative of Health: Public Health and the Regulated Body, Londres : Sage, 1995 ; LUPTON D. et PETERSEN A., The New Public Health: Health and Self in the Age of Risk, Londres : Sage, 1996.

${ }^{2}$ PINELL P., « Modern Medicine and the Civilizing Process », Sociology of Health and Illness, vol. $18, \mathrm{n}^{\circ} 1,1996$, p. $1-16$

${ }^{3}$ CLARKE A. et al. (dir.), Biomedicalization: Technoscience, Health, and Illness in the U.S., Durham : Duke University Press, 2010.

${ }^{4}$ NEAL A., « Michel Foucault », in EDKINS J. et VAUGHAN-WILLIAMS N. (dir.), Critical Theorists and International Relations, Londres : Routledge, 2009, p. 162-170.

${ }^{5}$ BRÖCKLING U., KRASMANN S. et LEMKE T. (dir.), Governmentality. Current Issues and Future Challenges, Londres : Routledge, 2011 ; BURCHELL G., GORDON C. et MILLER P. (dir.), The Foucault Effect: Studies in Governmentality, Chicago : University of Chicago Press, 1991 ; MILLER P. et ROSE N., Governing the Present. Administering Economic, Social and Personal Life, Cambridge : Polity Press, 2008; NADESAN M., Governmentality, Biopower, and Everyday Life, Londres : Routledge, 2008. 
Dans la mesure où Michel Foucault destine les notions esquissées ci-dessus à caractériser un très large ensemble de situations politiques, collectivement observables depuis environ trois siècles, ${ }^{6}$ il nous a paru intéressant de mettre ces notions à l'épreuve de l'analyse politique comparée. Les textes réunis dans ce dossier thématique se prêtent à cet exercice et présentent cinq cas de figure où la biopolitique participe à la construction d'un cadre d'analyse comparatif appliqué aux troubles dyslexiques (M. Woollven), aux risques biotechnologiques ( $\mathrm{N}$. Schiffino), aux inégalités de santé (C. Clavier), à la lutte contre le tabagisme (S. Guigner) et à la maladie d'Alzheimer (B. Lucas). À l'image d'initiatives antérieures menées en France et à l'étranger ${ }^{7}$, cet échantillon de recherches récentes vise à établir la fécondité empirique des notions foucaldiennes dans différents contextes de recherche, ici liés aux politiques de santé publique observables dans l'ensemble des démocraties occidentales.

La pratique de la comparaison, commune à l'ensemble des textes, donne son originalité particulière au dossier : l'analyse comparée s'y retrouve sous plusieurs angles, à commencer par la comparaison internationale, mais aussi infranationale et intersectorielle. Ces pratiques sont guidées par des cadres explicatifs liés à l'analyse des politiques publiques, qui fait fréquemment appel à la comparaison dans l'élaboration de ses stratégies de recherche ${ }^{8}$, et qui s'intéresse de plus en plus régulièrement aux problèmes de santé publique en lien avec l'irruption des «scandales sanitaires» dans l'action des gouvernements occidentaux. ${ }^{9}$ L'ensemble des travaux existants met toutefois peu l'accent sur la biopolitique comme concept opératoire de la comparaison entre des contextes sociaux et politiques distincts. Cette absence de dialogue entre biopolitique et comparaison est observable dans le travail de Michel Foucault lui-même, dans la mesure où ses recherches sur l'établissement de la médecine sociale en Allemagne, en Angleterre et en France négligent certaines des caractéristiques historiques et institutionnelles propres à chaque cas d'étude. ${ }^{10} \mathrm{Ce}$ souci comparatif supplémentaire aurait pourtant l'intérêt de montrer comment, dans des systèmes de santé et dans des contextes démographiques différents, une même rationalité politique du contrôle social par les | « comportements de vie» en vient graduellement à s'imposer aux gouvernants comme aux gouvernés sur le moyen terme. ${ }^{11}$

${ }^{6}$ Chez Michel Foucault, la notion de biopouvoir apparait initialement dans le premier tome de son Histoire de la sexualité (La volonté de savoir, Paris : Gallimard, 1976), mais ce sont surtout les cours qu'il donne au Collège de France de 1977 à 1979 (Sécurité, territoire, population et Naissance de la biopolitique, Paris: Gallimard, 2004) qui donnent à la biopolitique sa consistance intellectuelle. Dans ces textes et dans de nombreux autres, rassemblés dans Dits et écrits (Paris : Gallimard, 1991, 4 vol.), Foucault se donne une focale d'observation large commençant approximativement au XVII ${ }^{\mathrm{e}}$ siècle.

${ }^{7}$ MEYET S., NAVES M.-C. et RIBEMONT T. (dir.), Travailler avec Foucault. Retours sur le politique, Paris : L'Harmattan, 2005.

${ }^{8}$ LIMA L. et STEFFEN M., «Comparaisons internationales en politiques publiques : stratégies de recherche, méthodes et interprétation », Revue internationale de politique comparée, vol. $11_{2} n^{\circ} 3,2004$, p. 339-348.

${ }^{9}$ Pour deux exemples liés à la sécurité alimentaire et publiés dans un précédent dossier thématique sur les politiques de risque en Europe, cf. ALAM T., «Crises, acteurs politiques et changement institutionnel, un nouveau système de gestion des risques alimentaires en Grande-Bretagne ? » et BARBIER M., «Une interprétation de la constitution de l'ESB comme problème public européen », Revue internationale de politique comparée, vol. 10, $\mathrm{n}^{\circ} 2,2003$, p. 219-231 et p. 233-246 respectivement.

${ }^{10}$ RENAULT E., «Biopolitique, médecine sociale et critique du libéralisme », Multitudes, n³4, 2008, p. 195-205.

${ }^{11}$ LARSEN L. T., « The Birth of Lifestyle Politics. The Biopolitical Management of Lifestyle Diseases in the United States and Denmark », in BRÖCKLING U., KRASMANN S. et LEMKE T. (dir.), op. cit., p. 201-224. 
On peut rapidement rappeler quelques éléments de définition applicables à la biopolitique et à ses notions connexes. Fondamentalement, la biopolitique est un terme descriptif désignant l'ancrage des technologies libérales de gouvernement dans les propriétés biologiques des sujets. Ce phénomène s'observe dans la constitution de politiques de santé publique qui prêtent attention à la fois au contrôle collectif des populations et à la mise en discipline des comportements individuels. En conséquence, l'action des pouvoirs publics sous l'influence du libéralisme exprime une double métamorphose : d'une part, le pouvoir politique a modifié sa focale d'observation en la déplaçant de la mort vers la vie ; d'autre part, le pouvoir politique a modifié ses instruments d'action, qui abordent désormais le gouvernement des autres dans les mêmes termes que le « gouvernement de soi ». ${ }^{12}$ En d'autres termes, l'œuvre foucaldienne se concentre en large partie sur une évolution macro-historique de la rationalité gouvernementale, du répertoire ancien de la souveraineté au répertoire moderne du libéralisme.

Cette perspective diachronique, qui caractérise une large fraction des travaux de Michel Foucault au-delà des textes précités, nous semble mériter d'être empiriquement confrontée aux contextes contemporains des politiques de santé publique. Plusieurs niveaux de gouvernement se trouvent systématiquement impliqués dans la constitution de ces politiques. Un premier objectif de recherche peut consister à établir dans quelle mesure chaque niveau contribue à étendre le champ d'application de la biopolitique, et si l'articulation de ces niveaux de gouvernement peut être assimilée à un régime international de la santé politique. Ensuite, la biopolitique n'est pas un phénomène figé dans la « dialectique » initialement établie par Michel Foucault : le libéralisme a connu des développements, voire des dépassements, comme semblent l'indiquer les recherches menées sur le «libéralisme avancé »- ou sur le «néolibéralisme »- des États occidentaux. ${ }^{13}$ Un aspect particulièrement intéressant de la biopolitique contemporaine semble ainsi se situer dans son architecture rhétorique et opératoire à géométrie variable, oscillant entre l'autocontrôle et l'autoritarisme : la diffusion de messages incitant les individus à se distancier par eux-mêmes des comportements à risque se superpose aujourd'hui à des mesures prohibitives ou stigmatisantes de ces comportements. ${ }^{14}$ En conséquence, un deuxième objectif de recherche peut consister à réduire la diversité apparente des politiques de santé publique en les situant sur un continuum de pratiques rendues possibles par la sédimentation des initiatives gouvernementales.

Ces nuances dans l'application concrète de la biopolitique aux populations qu'elle est amenée à surveiller se comprennent d'autant mieux au moyen d'une approche comparative des politiques publiques, à laquelle la biopolitique peut fournir un point de départ conceptuel. En se donnant pour objectif général de comprendre les techniques politiques modernes de la subjectivation, notamment dans l'intention d'y résister, Michel Foucault nous semble en effet avoir élaboré un agenda de recherche authentiquement intersectoriel, permettant de rapprocher différents domaines de l'action publique à partir d'une conceptualisation de la rationalité poli-

\footnotetext{
${ }^{12}$ FOUCAULT M., L’herméneutique du sujet, Paris : Gallimard, 2001. Cette homologie explique le succès heuristique d'expressions foucaldiennes comme le « gouvernement à distance » ou la « conduite des conduites » dans l'analyse des appareils, dispositifs et technologies de gouvernement, que l'on regroupe ici dans la notion d'instrument pour faire écho à l'usage de ce terme par l'analyse des politiques publiques; LASCOUMES P., «La gouvernementalité : de la critique de l'État aux technologies du pouvoir », Le Portique, n¹3-14, 2004

${ }^{13}$ MILLER P. et ROSE N., Governing the Present. Administering Economic, Social and Personal Life, op. cit. ; JEANPIERRE L., "Une sociologie foucaldienne du néolibéralisme est-elle possible ? », Sociologie et sociétés, vol. 38, n², 2006, p. 87-111.

${ }^{14}$ Sur cette « sédimentation de dispositifs » gouvernementaux tournés pour partie vers l'autocontrôle, pour partie vers le stigmate et la pénalisation, cf. MEMMI D., «L'autoévaluation, une parenthèse ? Les hésitations de la biopolitique », Cahiers internationaux de sociologie, n¹28-129, 2010, p. 299-314.
} 
tique qui les traverse. ${ }^{15}$ Cette possibilité semble toutefois sous-exploitée dans la littérature scientifique, alors même que l'emploi fréquent du terme de "risques » ou de «facteurs de risque » dans le champ scientifique comme dans la rhétorique gouvernementale ${ }^{16}$ devrait inciter à mobiliser cette faculté de la notion de biopolitique. À cet égard, l'analyse politique comparée semble presque prédestinée à remédier à cet écueil, dans la mesure où ses objets et ses méthodes insistent sur l'hétérogénéité de l'action publique menée dans différents contextes politiques et institutionnels.

Ce dossier s'insère dans cet agenda de recherche en concentrant les moyens de l'enquête empirique sur les formes contemporaines de la biopolitique. Les problèmes publics étudiés dans chaque cas de figure ont, pour la plupart, une existence courte, voire très courte, et leur exposition est primordialement synchronique une fois effectué le rappel de leurs antécédents historiques. Cette perspective met en relief les régularités et les variations des phénomènes étudiés et les contextualise en les mettant en rapport aux institutions politiques et sociales qui façonnent l'action publique de manière durable. ${ }^{17}$ Une première partie des textes traite ainsi de la problématisation et de la politisation des politiques de santé publique, qui se construisent au contact des stratégies de légitimation des acteurs politiques (C. Clavier) et des controverses exprimant une demande de régulation de leur part (N. Schiffino). Une deuxième partie des textes traite ensuite des modes d'intervention déployés à travers ces politiques, lesquelles sont fortement structurées par les pratiques professionnelles de leurs agents (M. Woollven) et par les configurations institutionnelles préexistant à leur mise en œuvre (B. Lucas). L'éclatement des politiques publiques sur plusieurs niveaux de gouvernement, le recours à des instruments d'action publique moins coercitifs qu'incitatifs et la mobilisation se retrouvent enfin au niveau de l'Union européenne, où la biopolitique s'exprime également dans le rapport de forces entretenu entre la Commission européenne et les États-membres (S. Guigner).

Dans l'ensemble des textes réunis, la référence à Michel Foucault ne se substitue pas aux modèles explicatifs des auteurs : elle offre des «prises à l'imagination sociologique ${ }^{18}$ mais n'invite pas à faire l'économie d'une méthode comparative. Au contraire, chaque auteur ancre sa stratégie de recherche dans un cadre théorique plus large, où la biopolitique apparait avant tout comme une hypothèse d'arrière-plan. Cet angle d'approche amène chaque texte à souligner la diversité des situations étudiées et à suggérer, en conséquence, certains amendements à la notion initialement avancée par Foucault. Dans plusieurs textes, il est ainsi évident que le correctif théorique apporté par Didier Fassin au moyen de la notion de «biolégitimité » c'està-dire « la manière dont les problèmes sociaux trouvent, non pas leur solution, mais leur ex-

${ }^{15}$ À titre d'exemple, la notion de discipline, initialement avancée par Michel Foucault en rapport au champ pénal, se retrouve entièrement dans la notion plus large de « biopédagogie », qui s'applique en santé publique à des pathologies comme l'obésité ; HARWOOD V., " Theorizing Biopedagogies », in WRIGHT J. et HARWOOD V. (dir.), Biopolitics and the 'Obesity Epidemic': Governing Bodies, Londres : Routledge, 2009, p. 15-30. Le terme suggère aussi des recoupements possibles entre la santé publique et le champ éducatif.

${ }^{16}$ CASTEL R., La gestion des risques, Paris : Minuit, 1981 ; SKOLBEKKEN J.-A., «The Risk Epidemic in Medical Journals », Social Science and Medicine, vol. 40, n 3, 1995, p. 291-305; LUPTON D., Risk, Londres : Routledge, 1999 ; PERETTI-WATEL P. et MOATTI J.-P., Le principe de prévention. Le culte de la santé et ses dérives, Paris : Seuil, 2009.

${ }^{17}$ Ce déplacement de regard est en partie inspiré de la carrière scientifique du concept de répertoire d'action collective, étudié par Charles Tilly et Sidney Tarrow de manière diachronique, à des fins d'historicisation de leur objet de recherche, puis synchronique, à des fins de comparaison internationale dans plusieurs idéaux-types de régimes politiques ; PÉCHU C., "Répertoire d'action collective », in FILlEUlE O., MATHIEU L. et PÉCHU C. (dir.), Dictionnaire des mouvements sociaux, Paris : Presses de Sciences Po, 2009, p. 454-462.

${ }^{18}$ FABIANI J.-L., « La sociologie historique face à l'archéologie du savoir », Le Portique, n ${ }^{\circ} 13-14$, 2004. 
pression la plus autorisée dans le langage de la santé publique $»,{ }^{19}$ possède une vertu heuristique complémentaire aux intuitions originelles de la notion de biopolitique. La coopération entre acteurs publics et acteurs privés dans la mise en place de dispositifs biopolitiques mériterait aussi une attention supplémentaire à l'avenir, étant donné l'influence des partenariats observés dans le domaine de la santé publique. Enfin, l'extension de la biopolitique à d'autres secteurs que la santé publique est prévisible au regard des ambitions de ses agents, qui envisagent fréquemment d'appliquer le mot d'ordre de l'Organisation Mondiale de la Santé concernant l'extension de la santé à toutes les politiques. ${ }^{20}$ Parfois « impérialiste » dans son rapport aux autres rationalités gouvernementales, parfois «tyrannique » ${ }^{21}$ dans son rapport aux populations qu'elle surveille, la biopolitique n'apparaît ainsi plus comme une simple reconfiguration de l'action publique, mais comme une entreprise politique à part entière, portée par des acteurs en concurrence les uns avec les autres dans un contexte d'évolution constante des instruments de l'action publique et de ses objectifs. Le défi scientifique que constitue l'analyse de cet état de fait apparaît également comme une condition préalable à sa subversion, ou au moins à l'établissement de points de résistance, ce que Michel Foucault avait luimême identifié comme un point d'aboutissement possible de son œuvre intellectuelle.

Thibault Bossy est maître de conférences en sociologie à l'Université Bordeaux Segalen et chercheur au Centre Émile Durkheim. Ses recherches portent sur les politiques comparées de lutte contre l'obésité.

Coordonnées: Université Bordeaux Segalen, Département de sociologie, 3ter place de la Victoire, 33076 Bordeaux cedex; thibault.bossy@u-bordeaux2.fr.

François Briatte est doctorant en science politique à l'Institut d'Études Politiques de Grenoble, sous la direction de Monika Steffen. Ses recherches portent sur les politiques de lutte contre le cancer.

Coordonnées: Sciences Po, Courrier des personnels enseignants, 28 rue des Saints-Pères, 75007 Paris ;f.briatte@ed.ac.uk.

${ }^{19}$ FASSIN D., 1998. « Politique des corps et gouvernement des villes. La production locale de santé publique », in FASSIN D. (dir.), Les figures urbaines de la santé publique, Paris : La Découverte, p. 746 (cit. p. 40) ; FASSIN D., « La biopolitique n'est pas une politique de la vie », Sociologie et sociétés, vol. $38, \mathrm{n}^{\circ} 2,2006$, p. 35-48.

${ }^{20} \mathrm{KRECH}$ R. et al., «Implications of the Adelaide Statement on Health in All Policies », Bulletin of the World Health Organization, vol. 88, n¹0, 2010, p. 720.

${ }^{21}$ MEMMI D., op. cit., p. 313. 